\title{
Bilateral Transversus Thoracis Muscle Plane Block Provides Effective Analgesia and Enhances Recovery After Open Cardiac Surgery
}

Yang Zhang ( $\sim$ mzzhangyang@126.com )

First Affiliated Hospital of Nanchang University https://orcid.org/0000-0001-9674-4052

Haixia Gong

First Affiliated Hospital of Nanchang University

Biming Zhan

Second Affiliated Hospital of Nanchang University

Shibiao Chen

First Affiliated Hospital of Nanchang University

Research article

Keywords: The transversus thoracis muscle plane block, The length of hospital stay, Sufentanil, Numerical Rating Scale, Postoperative pain, Open cardiac surgery

Posted Date: December 4th, 2020

DOl: https://doi.org/10.21203/rs.3.rs-120187/v1

License: (9) This work is licensed under a Creative Commons Attribution 4.0 International License. Read Full License

Version of Record: A version of this preprint was published at Journal of Cardiac Surgery on May 28th, 2021. See the published version at https://doi.org/10.1111/jocs.15666. 
Bilateral Transversus Thoracis Muscle Plane Block Provides Effective Analgesia and Enhances Recovery After Open Cardiac Surgery

Yang Zhang ${ }^{1 *}$, MD, Haixia Gong ${ }^{1}$, MD, Biming Zhan ${ }^{2}$, MD, Shibiao Chen, MD ${ }^{1}$

*Correspondence author: Dr. Yang Zhang

1 Department of Anesthesiology, First Affiliated Hospital of Nanchang University, 17

Yong wai zheng Street, Nanchang, Jiangxi, 330006,China.

2 Department of cardiology, The second Affiliated Hospital of Nanchang University,

NO.1 minde Street, Nanchang, Jiangxi, 330006, China.

Tel: +8679188692556

Fax: +8675188692558

E-mail: mzzhangyang@126.com

Word count: 2989

Number of tables:2

Number of figure: 3 


\section{Abstract}

Background: The mid-sternum is the main source of pain after open cardiac surgery. The aim of this study was to investigate the effect of bilateral transversus thoracis muscle plane (TTMP) blocks on open cardiac surgery.

Methods: Sixty patients were randomly divided into two groups: bilateral TTMP blocks (TP group) or no nerve block (CO group). The primary endpoint was perioperative sufentanil consumption. The secondary outcome measures included postoperative pain, flurbiprofen axetil administration, quality of sleep after extubation, time to extubation, time to the return of gastrointestinal function, time to drain removal, the Intensive Care Unit (ICU) stay time and hospital stay.

Results: The TP group reported significantly less sufentanil and flurbiprofen axetil consumption than the $\mathrm{CO}$ group. The $\mathrm{CO}$ group had higher Numerical Rating Scale (NRS) pain scores at 1, 2, 6,12, $24 \mathrm{~h}$ after extubation both at rest and during movement than the TP groups. Compared to the $\mathrm{CO}$ group, time to extubation, time to the first bowel movement, ICU stay time and hospital stay were significantly decreased in the TP group. The TP group was rated as better in the quality of the two nights of sleep after extubation.

Conclusion: Bilateral TTMP blocks can provide good perioperative analgesia for patients undergoing open cardiac surgery and promote postoperative recovery. Trial registration: This study was registered in the Chinese Clinical Trial Registry (ChiCTR2000032318) on 04/08/2019. 
Key words: The transversus thoracis muscle plane block; The length of hospital stay; Sufentanil; Numerical Rating Scale ; Postoperative pain; Open cardiac surgery.

\section{Introduction}

Postoperative pain is severe in patients undergoing cardiac surgery, especially from the median sternotomy incision ${ }^{1,2}$. According to a previous study, 705 patients undergoing open cardiac surgery suffer from pain, scoring between 5.3 and 6.5 out of 1024 hours postoperatively ${ }^{2}$. The mid-sternum is the main source of pain after cardiac surgery ${ }^{3}$. The current analgesia regimen with the use of oral and intravenous analgesics has a limited effect. Poor postoperative analgesia in patients undergoing cardiac surgery increased morbidity and a longer hospital stay than patients without pain ${ }^{3,4}$. High-dose opioids can provide good postoperative analgesia for patients undergoing heart surgery. However, opioids has some side effects, such as nausea and vomiting, pruritus, and respiratory depression, as well as increased risk of chronic pain $^{5}$. Therefore, the implementation of neuraxial and paravertebral block techniques may be a superior choice ${ }^{6,7}$.

Paravertebral block and thoracic epidural anesthesia (TEA) result in significantly low pain scores after open cardiac surgery ${ }^{8}$. Epidural hematoma caused by postoperative coagulopathy and anticoagulation, hemodynamic instability and arterial or venous epidural puncture have limited the wide application of regional techniques in cardiac surgery patients ${ }^{9}$. The effect of continuous infusion of local anesthetics on postoperative analgesia in cardiac surgery patients is mainly related to the 
concentration of local anesthetics and the position of catheters, thus the application of this technology is limited ${ }^{10}$. So it seems that ultrasound-guided peripheral nerve block is the safest and the most effective method for postoperative analgesia in cardiac surgery patients.

The transversus thoracis muscle plane (TTMP) block is a newly developed technique ${ }^{11,12}$ that was first reported by Ueshima in $2015^{13}$. The TTMP block covers the anterior branches of intercostal nerves from $\mathrm{T} 2$ to $\mathrm{T} 6$ to provide effective analgesia in the internal mammary area ${ }^{14}$. Therefore, bilateral TTMP block may provide an effective analgesic alternative during median sternotomy in cardiac surgery patients. The purpose of this study was to observe whether bilateral TTMP blocks can provide good postoperative analgesia and promote postoperative rehabilitation for patients undergoing open heart surgery.

\section{Methods}

This randomized, double-blind study was approved by the ethics committee of our hospital, and it was registered in the Chinese Clinical Trial Registry

(ChiCTR2000032318). Our study adheres to CONSORT guidelines.

\section{Patients and design}

This study included patients aged 18 to 70 years, American Society of Anesthesiologists physical status II-III, who underwent median open heart surgery. The exclusion criteria were patient refusal, hepatic or renal failure, unable to cooperate and communicate, ejection fraction $<35 \%$, allergy to ropivacaine, secondary 
surgery, urgent surgery, hemodynamic instability and drug addiction.

\section{Surgery and anesthesia}

All patients received $200 \mathrm{ml}$ carbohydrate loading before entering the operating room.

General anesthesia was induced with $0.1 \mathrm{mg} / \mathrm{kg}$ midazolam, $0.3 \mathrm{mg} / \mathrm{kg}$ etomidate, $0.15 \mathrm{mg} / \mathrm{kg}$ cisatracurium and $0.6-1 \mu \mathrm{g} / \mathrm{kg}$ sufentanil. Then, endotracheal intubation was performed. Total intravenous anesthesia was maintained with propofol and cisatracurium, and the BIS was maintained between 45 and 55 . The administration of sufentanil during surgery was decided according to clinical need. According to the demands of the patients, postoperative analgesia was performed with continuous infusion of sufentanil. If patients complained of additional pain (NRS score $\geq 4$ ), $50 \mathrm{mg}$ flurbiprofen axetil was injected i.v. at $6 \mathrm{~h}$ intervals. No other analgesic drugs were used in any of the patients during the perioperative period.

The patients undergoing cardiac surgery were randomly divided into two groups after providing written informed consent. Patients in the TP group underwent bilateral TTMP blocks after endotracheal intubation, whereas in the other group (the CO group), no nerve block was performed (the same volume of saline was injected as the TTMP block in the experimental group). All operations were performed by the same group of surgeons, and all patients were sent to ICU.

\section{Randomization and blinding}

All enrolled patients were randomly divided into TP group or CO group with either $0.4 \%$ ropivacaine or saline for using a computer generated random number table, and the group allocation was kept in the sealed envelope. The saline and ropivacaine were 
prepared in the post anesthesia care unit by a nurse, the saline and ropivacaine solutions looked identical. The skilled anesthesiologist injected the liquid into TTMP within 20 min and he didn't know whether the liquid is ropivacaine or saline.

Postoperative data collection was recorded by another researcher. The patients, surgeons, anesthesiologist, intensive care unit staff, nurses and other investigators were not aware of medication assignment. Thus this was a double-blind, randomized, controlled study.

\section{Ultrasound-guided TTMP block}

We used a real-time high-frequency linear ultrasound probe (Huasheng, Shenzhen, China) to perform bilateral TTMP blocks. After using ultrasonography to determine the anterior T4-T5 interspace, the ultrasound probe parallel to the rib was placed lateral to the sternal border ${ }^{15}$ so that we could find the pectoralis major internal muscle, intercostal muscle and transversus thoracis muscle. The TTMP was located between the two posterior muscles. The internal mammary artery and vein also passed through the TTMP, verifying this plane. Then, a 20-gauge, $70 \mathrm{~mm}$ needle (Tuoren, Henan, China) was inserted inline with the tip of the needle located in the TTMP, and $0.4 \%$ ropivacaine $(20 \mathrm{ml})$ was injected into this plane. After 5 minutes, we used ultrasonography to observe whether the local anesthetic had spread between the costal cartilage and the transversus thoracis muscle in T2-6. If ropivacaine had spread poorly, we added local anesthetic in the corresponding plane to ensure an analgesic effect. The methods used on the other side of the TTMP blocks were the same. All nerve blocks were completed by the same skilled anesthesiologist within 15 minutes. The 
possible complications of TTMP blocks in this study included ropivacaine allergy, pneumothorax, hematoma, infection and injury of the internal mammary artery and vein. The TTMP block was done as previously described ${ }^{16}$.

\section{Study Parameters}

The primary endpoint was perioperative sufentanil consumption. The secondary outcome measures included pain at rest and during coughing (exercise pain) at 1, 2, 6, 12,24 , and $48 \mathrm{~h}$ after extubation ;48-hour flurbiprofen axetil administration; quality of the two nights' of sleep after extubation; time to extubation; time to return of gastrointestinal function (including the first bowel movement and the first occurrence of flatus); time to drain removal; the Intensive Care Unit (ICU) stay time and hospital stay.

Postoperative pain was measured using the Numerical Rrating Scale (NRS) score from 0 (no pain) to 10 (worst severe pain). The sleep quality assessment was performed with a $10-\mathrm{cm}$ visual analogue scale $(0=$ worst sleep quality, $10=$ best sleep quality). Data collection was recorded by an experimental assistant who was also blinded to the experimental grouping.

\section{Statistical analysis}

The calculation of the patient sample size was based on a pilot study ( $\mathrm{n}=8$ patients in each group), which compared the primary endpoint, i.e., perioperative sufentanil consumption during open cardiac surgery. A sample size of 25 patients in each group was required with a type I error of $\alpha=0.05$, a type II error of $\beta=0.1$ and a power of 
$90 \%$. Considering the possible surgical reasons for exclusion and the possibility of patient drop out during the study, we included $20 \%$ additional patients for the final sample size ( $\mathrm{n}=30$ in each group).

Pain intensity after extubation was compared between the TP group and $\mathrm{CO}$ group with repeated-measures (two-way) analysis of variance. Student's t test was used to assess intergroup differences with a normal distribution, whereas the Wilcoxon Mann-Whitney test was used to assess abnormally distributed data. A probability value of less than $5 \%$ was considered significant.

\section{Results}

Sixty-six patients were enrolled in our trial. Six patients were excluded for the following reasons: secondary surgery (two); ejection fraction $<35 \%$ (two) and renal failure (two). Ultimately, a total of 60 patients were included in our study for data analysis, with 30 in each group (Fig 1). No differences in patients' characteristics or other factors were noted between the groups (Table 1).

The TP group required significantly less intraoperative and postoperative sufentanil consumption than the CO groups (Table 2). The CO group had higher NRS pain scores than the TP group at 1, 2, 6, $24 \mathrm{~h}$ after extubation both at rest and during movement; time points both at rest and during movement (Fig 2, Fig 3). Patients in the $\mathrm{CO}$ group received significantly more flurbiprofen axetil in the first 48 hours than patients in the TP group (Table 2). The time to extubation, time to the first flatus, length of stay in the ICU and length of hospital stay were significantly decreased in 
the TP group compared with the CO groups (Table 2). The quality of the two nights' of sleep after extubation was rated as better in the TP group than in the CO group (Table 2). There were no significant differences between the groups in terms of the time to first feces or the time to drain removal (Table 2). No complications due to the TTMP blocks occurred in our study.

\section{Discussion}

The present study demonstrated that the use of ultrasound-guided TTMP blocks could reduce the perioperative sufentanil consumption, dosage of postoperative flurbiprofen axetil, time to extubation, time to the first flatus, length of stay in the ICU and length of hospital stay in patients undergoing open cardiac surgery. Furthermore, the TTMP block also provide effective analgesia and good sleep quality for patients who underwent open cardiac surgery.

The use of TTMP blocks has been reported in recent years ${ }^{11,12}$; this type consists of a shallow block, and there were no adverse events in our study, such as hematoma, arterial puncture, ropivacaine allergy, pneumothorax or infection. A previous study that included 299 patients who underwent TTMP blocks showed that there were only 2 incidences of 'slight infections' around the injection site ${ }^{17}$. Therefore, TTMP blocks represent a safe technique that can be widely used in patients who undergo open cardiac surgery ${ }^{18}$. Ueshima et al ${ }^{15}$ found that the spread of ropivacaine in the TTMP between the fourth and fifth ribs was larger than that between the third and fourth ribs. Therefore, the fourth and fifth ribs next to the sternum were used in all patients in our 
study for the TTMP block.

High-dose sufentanil was able to maintain hemodynamic stability; it is still widely used in many countries for bolus dosing or infusion regimes during cardiac anesthesia $^{19}$. However, high-dose opioid techniques cause PONV, respiratory depression, pruritus, delayed recovery, prolonged ventilation, increased ICU stays, increased costs ${ }^{20}$, and limited postoperative recovery after open cardiac surgery ${ }^{21}$. Our study revealed that the use of bilateral TTMP blocks decreased the consumption of perioperative sufentanil without adverse events and that they may work via the following two aspects. On the one hand, the TTMP block was found to cover the T2-T6 intercostal nerves ${ }^{11}$, and it had the potential to provide analgesia for surgery of the anterior chest wall. Therefore, it could greatly reduce the amount of intraoperative sufentanil used during open cardiac surgery before opening the sternum. On the other hand, this block reduced the dose of postoperative sufentanil needed by providing effective postoperative analgesia ${ }^{22}$. Therefore, reducing the perioperative dose of sufentanil was an important part of the enhanced recovery of cardiac surgery.

Open cardiac surgery results in severe and prolonged postoperative pain, especially at the median sternotomy site, and peaking during the first two days after the operation $^{23}$. Poorly controlled postoperative pain in open cardiac patients results in hemodynamic instability, hypercoagulability, pulmonary complications, sympathetic activation, and increased rates of delirium ${ }^{24}$.In the current study, we demonstrated that bilateral TTMP blocks provided effective analgesia during open cardiac surgery both at rest and during mobilization. Moreover, sufentanil and flurbiprofen axetil 
consumption was still significantly lower in the TP group than in the $\mathrm{CO}$ group during the $48 \mathrm{~h}$ after surgery, and it could reduce the adverse effects of these two drugs. Neuraxial techniques and paravertebral nerve blocks can also provide effective postoperative analgesia for patients undergoing open cardiac surgery ${ }^{25}$. However, they are not routinely performed in cardiac surgery due to concerns of hemorrhage and hematoma after coagulopathy and heparinization ${ }^{24}$. Therefore, ultrasound-guided TTMP blocks represent a novel, effective, promising, and safe regional analgesic technique in cardiac surgery and should be widely used.

Sleep quality is critical for patients' postoperative comfort and fatigue after cardiac surgery. Our results showed that the TP group had better sleep quality than the CO group, which may be associated with the enhanced recovery after cardiac surgery. Better pain relief and pain reduction after sufentanil consumption contributed to better sleep quality in the TP group because sufentanil is known to disrupt sleep quality ${ }^{26}$.Our study demonstrated that bilateral TTMP blocks led to early extubation and early time to the first bowel movement. Finally, decreased sufentanil consumption, improved pain control, early extubation and early time of the first flatus were responsible for the reduction in the length of stay in the ICU and the length of hospital stay.

This study has some limitations. The volume and concentration of the TTMP block used in our study was based on previous research. In further trials, the optimum capacity and concentration of the TTMP block in open cardiac surgery should be evaluated. Continuous TTMP block may provide optimal and long postoperative 
analgesia during median sternotomy ${ }^{27}$, but our study did not use this technique. Therefore, randomized controlled studies are necessary to explore the utility of continuous TTMP block in different surgical patients. In addition, effective postoperative pain relief may prevent the development of chronic pain ${ }^{28}$, but we did not observe the effect of this block on postoperative chronic pain.

In conclusion, this study showed that the use of ultrasound-guided TTMP block in open cardiac surgery reduced the length of hospital stay by providing effective postoperative pain, reducing sufentanil and flurbiprofen axetil consumption, decreasing the mechanical ventilation time and the time to the first flatus, improving sleep quality and reducing the length of stay in the ICU.

\section{Declarations}

\section{Ethics approval and consent to participate}

This study was approved by the First Affiliated Hospital of Nanchang University (Ethical Committee number 201926; Chairperson Ge Gao) and registered in the Chinese Clinical Trial Registry (ChiCTR2000032318) on 04/08/2019.Written informed consent was obtained from each patient.

\section{Consent for publication}

Not applicable.

\section{Availability of data and materials}

The datasets used during the current study are available from the corresponding author on reasonable request.

\section{Competing interests}

The authors declare that they have no competing interests or disclosures.

\section{Funding}

The authors received no external funding.

\section{Authors' contributions}


$\mathrm{YZ}$ and SBC were resposible for conceived, designed this study and collected the data. $\mathrm{YZ}$ and BMZ were responsible for study execution and manuscript writing. HXG was responsible for data analysis. All authors have read and approved the final version of the manuscript.

\section{Acknowledgements}

None.

\section{References}

1 Lahtinen P, Kokki H, Hynynen M. Pain after cardiac surgery: A prospective cohort study of 1-year incidence and intensity. Anesthesiology 2006;105:794-800.

2 Milgrom LB, Brooks JA, Qi R, et al. Pain levels experienced with activities after cardiac surgery. Am J Crit Care 2004;13:116-25 .

3 Mueller XM, Tinguely F, Tevaearai HT, et al. Pain location, distribution, and intensity after cardiac surgery. Chest 2000;118:391-6.

4 Bigeleisen PE, Goehner N. Novel approaches in pain management in cardiac surgery. Curr Opin Anaesthesiol 2015;28:89-94.

5 Fletcher D, Martinez V. Opioid-induced hyperalgesia in patients after surgery: A systematic review and a meta-analysis. Br J Anaesth 2014;112:991-1004.

6 Carli F, Kehlet H, Baldini G, et al. Evidence basis for regional anesthesia in multidisciplinary fast-track surgical care pathways. Reg Anesth Pain Med 2011;36:63-72.

7 Andreae MH, Andreae DA. Regional anaesthesia to prevent chronic pain after surgery: A Cochrane systematic review and meta-analysis. Br J Anaesth 2013;111:711-20.

8 Hemmerling TM, Prieto I, Choiniere JL, et al. Ultra-fast-track anesthesia in off-pump coronary artery bypass grafting: A prospective audit comparing 
opioid-based anesthesia vs thoracic epidural based anesthesia. Can J Anesth 2004;51:163-8.

9 Ho AM, Chung DC, Joynt GM. Neuroaxial blockade and hematoma in cardiac surgery: Estimating the risk of a rare adverse event that has not yet occurred. Chest 2000;1117:551-5.

10 Jagannathan R, Niesen AD, D'Souza RS, Johnson RL. Intermittent bolus versus continuous infusion techniques for local anesthetic delivery in peripheral and truncal nerve analgesia: the current state of evidence. Reg Anesth Pain Med.2019 $04 ; 44(4): 447-451$

11 Ueshima H, Takeda Y, Ishikawa S, et al. Ultrasound-guided transversus thoracic muscle plane block: a cadaveric study of the spread of injectate. J Clin Anesth 2015;27:696.

12 Ueshima H, Kitamura A. Blocking of multiple anterior branches of intercostal nerves (Th2-6) using a Transversus thoracic muscle plane block. Reg Anesth Pain Med 2015;40:388.

13 Ueshima H, Kitamura A. Clinical experiences of ultrasound-guided transversus thoracic muscle plane block: a clinical experience. J Clin Anesth 2015;27:428-9.

14 Ueshima H, Hara E, Marui T, et al. The ultrasound-guided transversus thoracic muscle plane block is effective for the median sternotomy. J Clin Anesth 2016;29:8.

15 Ueshima $\mathrm{H}$, Otake $\mathrm{H}$. Where is an appropriate injection point for an ultrasound-guided transversus thoracic muscle plane block? J Clin Anesth 2016;33:190-1. 
16 Zhang, Y; Chen, S; Gong, H; Zhan, B. Efficacy of Bilateral Transversus Thoracis Muscle Plane Block in Pediatric Patients Undergoing Open Cardiac Surgery. J Cardiothorac Vasc Anesth. J Cardiothorac Vasc Anesth 2020 Sep;34(9):2430-2434.

17 Ueshima H, Otake H. Ultrasound-guided transversus thoracic muscle plane block: complication in 299 consecutive cases. J Clin Anesth 2017;41:60.

18 Satoru Fujii, Matthew Roche, Philip M Jones, et al. Transversus thoracis muscle plane block in cardiac surgery: a pilot feasibility study. Reg Anesth Pain Med 2019;44:556-560.

19 Kwanten LE, O'Brien B, Anwar S. Opioid-Based Anesthesia and Analgesia for Adult Cardiac Surgery: History and Narrative Review of the Literature. J Cardiothorac Vasc Anesth.2019 Mar ;33(3):808-816

20 Lena P, Balarac N, Lena D, et al. Fast-Track Anesthesia With Remifentanil and Spinal Analgesia for Cardiac Surgery: The Effect on Pain Control and Quality of Recovery. J Cardiothorac Vasc Anesth 2008;22:536-42.

21 Mazzeffi M, Khelemsky Y. Poststernotomy pain: a clinical review.J Cardiothorac Vasc Anesth. 2011;25:1163-78.

22 Fujii, S. Transversus thoracis muscle plane block and alternative techniques. Reg Anesth Pain Med.2019 Jul 11 .

23 Thabane L, Ma J, Chu R, et al. A tutorial on pilot studies: the what, why and how. BMC Med Res Methodol 2010;10:1.

24 A. P. Huang and R. K. Sakata, "Pain after sternotomy - review," Revista Brasileira de Anestesiologia 2016; 66:395-401,

25 E. Bignami, A. Castella, V. Pota et al., "Perioperative pain management in cardiac surgery: a systematic review,” Minerva Anestesiologica 2018; 84: 488-503. 
26 Keifer J, Baghdoyan H, Lydic R: Sleep disruption and increased apnea after pontine microinjection of morphine. Anesthesiology 1992; 77:973-82.

27 Ueshima H,Otake H. Continuous transversus thoracic muscle plane block is effective for the median Sternotomy. J Clin Anesth.2017 $02 ; 37: 174$.

28 Kairaluoma PM, Bachmann MS, Rosenberg PH, Pere PJ. Preincisional paravertebral block reduces the prevalence of chronic pain after breast surgery. Anesth Analg 2006; 103:703-8. 


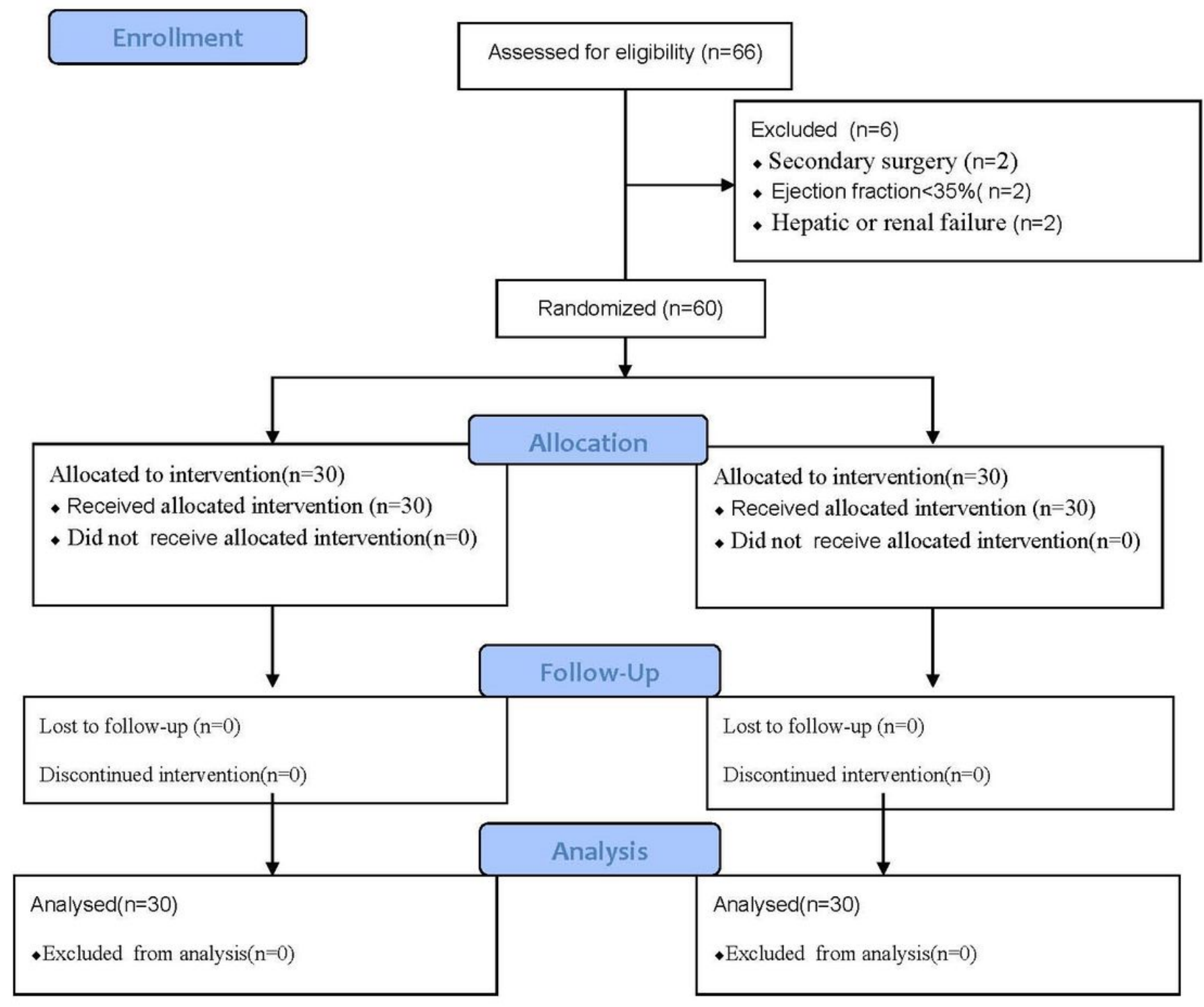

\section{Figure 1}

Patient flow diagram 


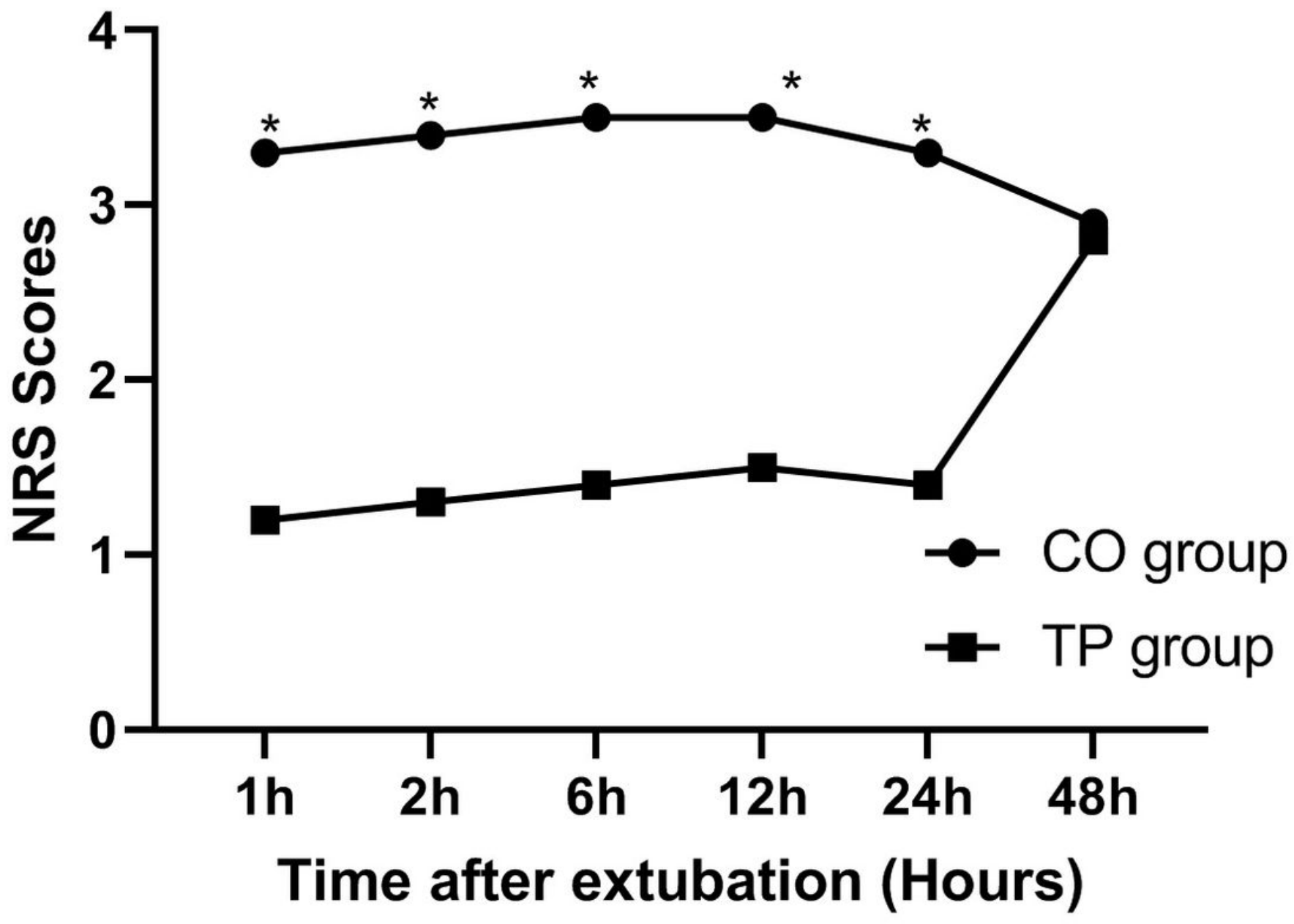

Figure 2

Pain intensity at rest after extubation which was measured using the verbal numerical scale (NRS) score. * $\mathrm{P}<0.05$ considered statistically significant. Results are mean \pm SD. 


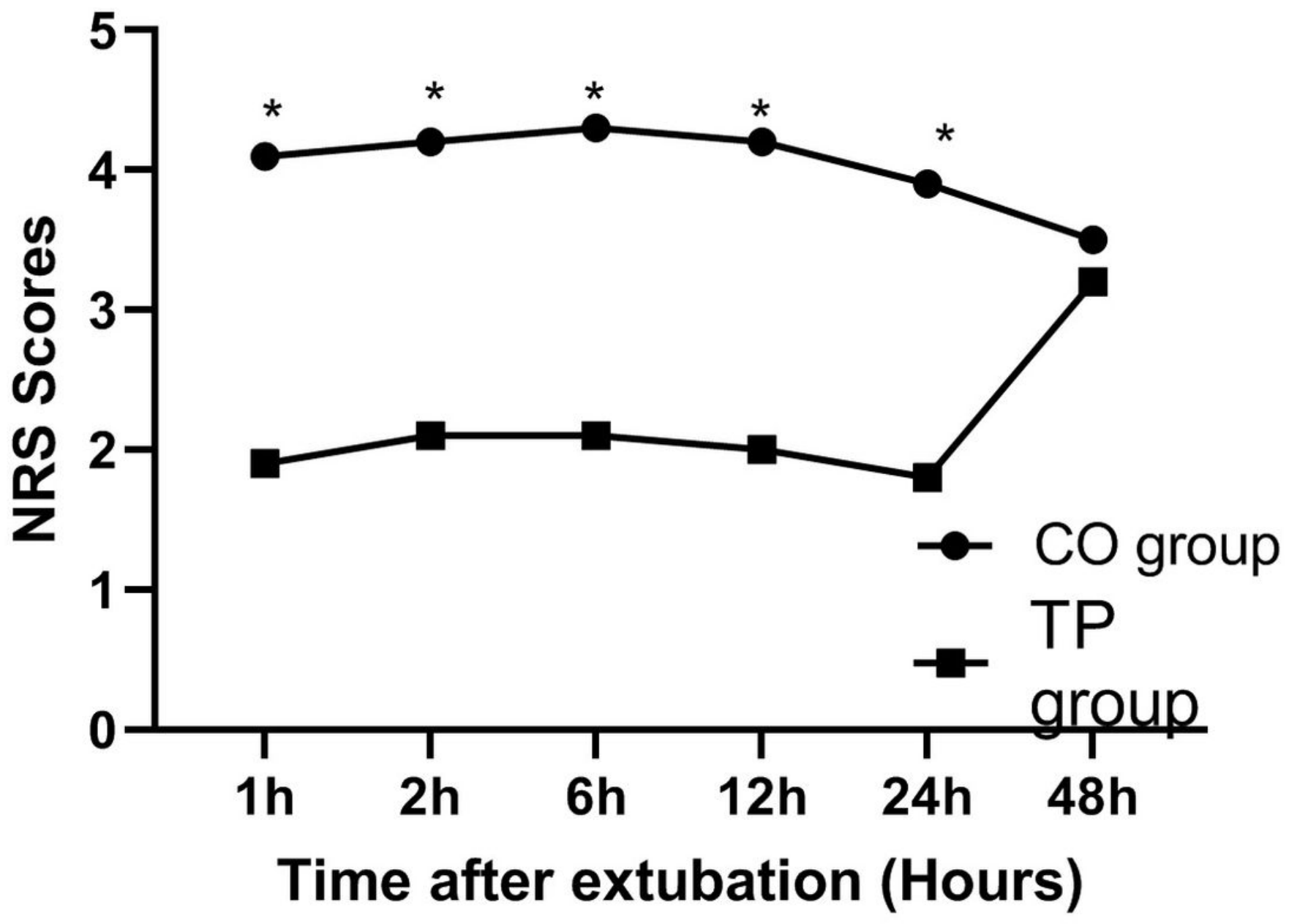

Figure 3

Pain intensity at movement after extubation which was measured using the verbal numerical scale (NRS) score. * $\mathrm{P}<0.05$ considered statistically significant. Results are mean $\pm \mathrm{SD}$.

\section{Supplementary Files}

This is a list of supplementary files associated with this preprint. Click to download.

- Table.doc

- CONSORT2010Checklist.doc 